\title{
Risk Analysis of the Impact of Metro Construction Activities on Cultural Relics
}

\author{
Xin Yunxiao $^{1}$, Li Xiaozhao ${ }^{1, *}$, Wang Jing ${ }^{2}$ \\ ${ }^{1}$ School of Earth Sciences and Engineering, Nanjing University, Nanjing 210023, China \\ ${ }^{2}$ College of Economics \& Management, Northwest A\&F University, Yangling 712100, China \\ hapoaaa@126.com \\ 地铁建设活动对文物的影响风险分析 \\ 辛皿潚 $^{1}$, 李晓昭 ${ }^{1, *}$, 王静 ${ }^{2}$ \\ 1 南京大学地球科学与工程学院, 南京 210023 , 中国 \\ 2 西北农林科技大学经济管理学院, 杨凌 712100 , 中国
}

hapoaaa@126.com

\begin{abstract}
With the expansion of urbanization scale, more and more infrastructures are being rebuilt in downtown areas. A majority of metropolises in China, however, have numerous cultural relics and historic sites. This situation has hindered redevelopment of infrastructures to some extent. As an important component in urban fabrics, metro development project can relief urban traffic pressure while causing less impact on the surface environment. In order to study the impact of urban subway construction projects and the influence of their operations on cultural and historical relics, this paper used cases of metro projects in cities of Beijing and Xi'an as examples, for following research tasks and objectives: 1) to classify cultural and historic relics, and to analyze their characteristics and protection requirements based on classification; 2) to study main influences of subway engineering to cultural relics during the underground construction process; 3) to analyze the negative effects of subway train vehicles on cultural relics during the operation process. Results showed that there are different influences of subway engineering on different types of culture relics. Land subsidence is the main factor affecting cultural relics during the construction process; while vibration generated by subway train vehicle during operation process, is the main negative effect for cultural relics and historical buildings.
\end{abstract}

Keywords-Subway development, Underground space, Historic structure, Land subsidence, Train vibration

摘要一随着城市化规模的扩大, 城市中心区基础设施的改 造越来越多。然而, 中国多数城市都有大量的文物古迹。这种 情况在一定程度上阻碍了基础设施的再开发。地铁开发项目作 为城市结构的重要组成部分, 在缓解城市交通压力的同时, 对 地表环境的影响较小。为了研究城市地铁建设项目的影响及其 运营对文物的影响, 本文以北京、西安等地的地铁项目为例, 进行以下研究任务和目标: 1) 对文物进行分类, 根据分类情况, 分析文物的特点和保护要求; 2) 研究地铁工程在地下施工过程 中对文物的主要影响; 3) 分析地铁车辆在运营过程中对文物的 负面影响。结果表明, 地铁工程对不同类型文物有不同的影响。

*通讯作者。
地面沉降是施工过程中影响文物的主要因素, 地铁车辆在运行 过程中产生的振动是文物和历史建筑的主要负面影响。

\section{关键词一地铁开发，地下空间，文物建筑，地面沉降，列车 振动}

\section{I. 引言}

随着城市的发展, 其规模不断增大, 城市人口密度也 在不断增加, 导致城区的交通压力也愈加严重。为缓解 城市交通设施的供需矛盾，市中心尤其是老城区地带亟 需新建交通设施以缓解交通压力。但老城区内的既有建 筑对交通设施的规划设计造成了一定的影响, 部分文物 需要严格保护, 因此许多地面设施的开发受限。地下交 通设施的开发可以较好地解决老旧城区的交通矛盾, 地 铁即是典型的城市地下交通设施之一。

然而, 影响较小并不代表地下设施的开发对地表建筑 完全没有扰动，地铁线路的规划设计仍需密切关注对地 表建筑尤其是文物古迹的影响。我国悠久的历史决定了 国内大部分城市保有数量可观的文物古迹, 因此城市的 开发需加强对其的保护。清华大学的肖金亮单就我国的 历史文物建筑的保护体系进行了系统的分析 ${ }^{[1]}$ 。涉及到地 下工程的影响, 长安大学的苗永杰就盾构法开挖对地表 文物的影响及其保护措施进行了相应的分析 ${ }^{[2]}$ 。具体到工 程案例而言, 西安地铁工程是较为典型的文物保护案例, 例如 2 号线在环城路内先后穿越了明城墙安远门、钟楼以 及永宁门三处明代建筑以及护城河 ${ }^{[3]}$, 整条线路所涉及到 的文物分布密度较大。针对该处典型文物保护案例, 杨 开忠就具体的影响因素以及相应的保护措施进行了研究 ${ }^{[4]}$, 西安建筑科技大学的狄文莉和常海青则就此建立了相应 的评估体系, 进一步指导评价设计施工过程中的文物保 护工作 ${ }^{[5,6]}$ 。

地铁建设对文物的影响主要表现在两方面，分别是施 工开挖过程中所造成的地表沉降以及运营过程中列车运 行所产生的振动。地下工程施工所造成的地面沉降方面, 王帆等人分析了大断面公路隧道在黄土地层开挖过程之 中的沉降变形规律 ${ }^{[7]}$, 颜治国则就地铁施工对黄土地层沉 
降所造成的影响进行了系统分析, 并讨论了处理应对方 案 ${ }^{[8]}$; 软土地层方面, 龚江飞和钟铮分别就天津和上海地 区软土沉降的以及对地标建筑的影响进行了分析 ${ }^{[9,10]}$ 。地 铁车辆振动方面, 孟昭博和钱春宇等人就西安地铁工程 中列车的振动对地表文物建筑的影响进行了分析 ${ }^{[11,12]}$, 贾颖绚等人则分析了北京地铁列车振动荷载对文物的影 响, 并提出了相应减少影响的措施 ${ }^{[13]}$ 。

就文物建筑保护而言，历史建筑与地铁隧道工程之间 的冲突需要得到长期妥善处理。因此, 在分析地铁对历史 建筑可能带来的不良影响后, 迫切需要提出一种互利的 途径。众所周知, 盾构法是地铁等地下工程的主要施工 方法。然而, 地下隧道不可避免地会引起不同程度的地 面向开挖方向移动, 并导致地表沉降 ${ }^{[14]}$ 。近年来国内外 众多学者对盾构隧道的沉降进行了大量的预测研究 ${ }^{[15,16]}$ 将现有结构和环境的地面沉降可能产生的不利影响降至 最低的一个基本要素是预测地面沉降剖面的能力 ${ }^{[17]}$ 。通 常, 地表沉降可采用经验或半经验方法、分析方法和数 值方法进行估算 ${ }^{[18]}$ 。本文以西安城墙为例, 采用三维数 值模拟方法对地铁施工引起的沉降进行评价。

除了沉降之外，振动还破坏了地铁运营期间的历史建 筑。地铁诱发振动是近年来研究的热点问题。Krylova 和 Ferguson（1994）建立了一个分析模型, 研究准静态荷载 通过轨道和枕木传播到土壤中的影响 ${ }^{[19]}$ 。另一方面, 尽 管计算方法繁琐, 计算时间长, 计算资源大, 但基于有 限元方法的数值模型常常被用于预测地铁诱发振动 ${ }^{[20]}$ 。 在有限元函数的推广之后, 许多改进的数值模型被广泛 地应用。例如, Hall (2003) 运用有限元模型来预测瑞典 高速铁路中的铁路诱发振动, 然后将结果与现场测量结 果进行比较 ${ }^{[21]}$ 。此外, 有限元法也很适合模拟隧道中地 铁产生的振动, 振动通过土壤传播, 并影响附近敏感建 筑物和基础设施的地基。因此, 对地铁引起的振动进行 检测和预测, 可以有效地保护建筑物, 尤其是历史文物古 迹。本文就地铁振动对北京万松老人塔的影响进行了实 例研究, 旨在为此类研究提供参考。

\section{II. 施工阶段对文物的影响}

（一）理论分析

盾构隧道施工的优点在于尽可能平稳迅速地完成施工, 且尽量减少对建筑物和地基中埋置材料的影响。但是, 盾 构施工也会引起地层松动和沉陷。因此, 隧道附近的周围 结构可能会出现一些变形、沉降或位移, 甚至不可逆转的 损坏。从本质上讲, 是地层的变形引起了附近构造的变形。 必须认识到, 只有控制地层才能避免建筑物的沉降变形。

由于盾构施工引起隧道周围地层的松动和沉降, 从视 觉上表现为地表沉降。因此, 隧道附近的结构将发生变形、 沉降或位移, 结构将受到损坏或破坏。周围结构的变形实 质上也是地层变形引起的。因此, 只有控制变形量, 才能 更好地控制周围结构的沉降变形。

在软土层中采用盾构施工时, 隧道横向所产生的地表 变形范围受隧道的埋深和其所处的地质土层状况影响较 大, 基本上接近土的破坏棱体范围。盾构施工在横向产生 的沉降量近似于 peck 提出的沉陷槽形状, 即正态分布曲 线, 隧道中心产生的沉降量最大, 地面沉降量随着离隧道 中心线距离的增加而逐渐减小。对于盾构法施工引起的地 面沉降预测，一般可采用 Peck 公式计算 ${ }^{[22]}$ :

$$
\delta_{2}(y)=\delta_{2 \max } \exp \left(-\frac{y^{2}}{2 i^{2}}\right)=\frac{V_{s}}{\sqrt{2 \pi i}} \exp \left(-\frac{y^{2}}{2 i^{2}}\right)
$$

式中: $\delta_{2}(y)$ 为距开挖隧道中心线横向距离为 $y$ 处某 点的坚向沉陷值;

$\delta_{2 \max }$ 为最大沉降量;

$y$ 为距离隧道中心线的距离;

$i$ 为沉降槽宽度系数

$$
i=\frac{h+a}{\sqrt{2 \pi} \operatorname{tg}\left(45^{\circ}-\frac{\varphi}{2}\right)} ;
$$

$\phi$ 为土的内摩擦角;

$h+a$ 为地面至隧道底面中心深度;

$V_{s}$ 为盾构单位长度的地层损失, $V_{s}=\frac{V_{i} \pi r_{0}^{2}}{4}$;

$V_{i}$ 为地层体积损失率;

$r_{0}$ 为盾构机外径

根据计算, 可认为地面沉降曲线反弯点以内的范围影 响较大, 反弯点至沉降曲线边缘的范围影响较小, 沉降曲 线以外的范围可认为无影响。peck 沉降槽宽度基本在 $20 \sim 30 \mathrm{~m}$ 范围内, 即隧道中心线 $10 \sim 15 \mathrm{~m}$ 范围内, 根据大 量地铁的施工监测数据, 盾构施工产生的地 面沉降量大 于 $10 \mathrm{~mm}$ 的情况基本发生在隧道中心线 $10 \mathrm{~m}$ 以内, 对于隧 道中心线 $10 \mathrm{~m}$ 以外的地面, 盾构施工产生的沉降量基本小 于 $10 \mathrm{~mm}$ 。

\section{（二）工程案例}

西安是中国著名的历史文化名城，位于关中平原的中 心地带, 是中国十三朝古都, 因此拥有大量的历史遗迹, 其中西安城墙因其 600 年的历史文化价值而受到高度保护。 墙体采用砖砌体结构夯实, 城墙剖面如图 1 所示。南门高 11.6 米, 宽 16-18 米, 北门高 12 米, 宽 16-18 米, 西安地 铁 2 号线采用盾构隧道施工方式, 穿越西安城墙、钟楼等 几座历史建筑下方地层。在一定范围内控制沉陷, 确保历 史建筑的安全是十分重要的。

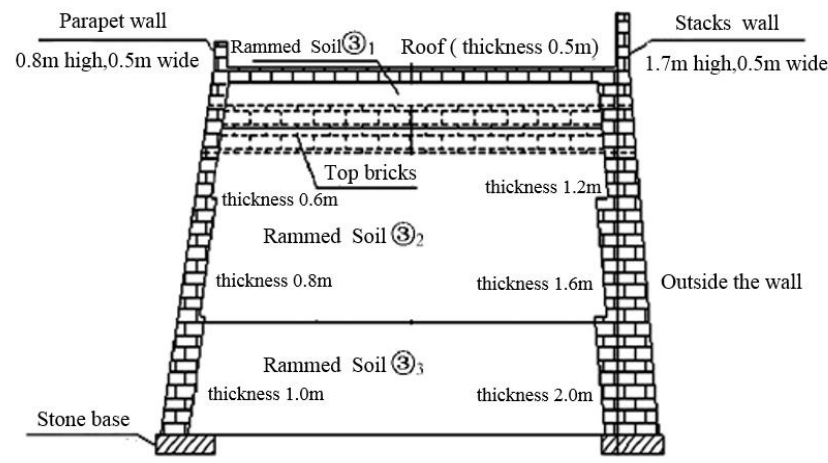

图 1. 西安城墙结构剖面图

（1）收集在建地铁资料，参考国内外地面沉降和建筑 物沉降控制标准。

（2）采用有限元方法模拟了城市墙体对沉降的响应。 通过建立隧道、基础、建筑物的数值分析模型, 可以得到 地铁施工时的城墙变形和应力状态。此外, 估算墙体的极 限状态, 生成沉降槽曲线, 其中最大沉降值为相应的城市 墙体变形极限值。因此, 南门与北门之间的地表沉降应控 
制在该限值范围内, 考虑到历史保护, 还应增加一定的安 全储备。应力矢量图和沉降槽矢量图见图 2(a) 和 (b)。 计算结果如图 4 所示。综上所述, 如果地面沉降控制在 -20 +5 mm（“+”表示隆起, “-”表示下沉)，那么城 墙结构足够安全。

（3）西安城墙历经近 600 年，在砖砌体中不可避免 地存在着沉降缝和夯土。研究这些节点的倾斜值, 有助于 了解整体发展趋势, 为沉降变形控制标准提供参考。

因此，沉降变形控制标准可制定如下：隧道在地下进 行时, 在南北大门范围内, 地表沉降应限制在

$-20 \mathrm{~mm}+5 \mathrm{~mm}$ 范围内，局部倾斜值应保持在 0.001 以内。 对于钟楼，地铁线路在该建筑物下有一定的避让空间，因
此沉降应在 $-5 \mathrm{~mm} \sim+5 \mathrm{~mm}$ 范围内变化, 局部倾斜值不大于 0.0005 。

（三）数值模拟计算预测

利用FLAC3D 建立了该段南大门沉降变形的三维数值 模型。该软件采用有限差分法, 在岩土工程分析领域和隧 道建模中得到了广泛应用 ${ }^{[22,23]}$ 。由于沉降缝分布的随机性, 为了简化模型, 本次计算没有建立沉降缝模型, 而是用安 全储备来代替，不同工况下的沉降变形都是基于不同的工 况计算的，如不同的地形条件下的沉降变形、半径、不同 埋深、不同加固方案, 以确定最佳施工方案。沉降变形云 图如图 3 所示。
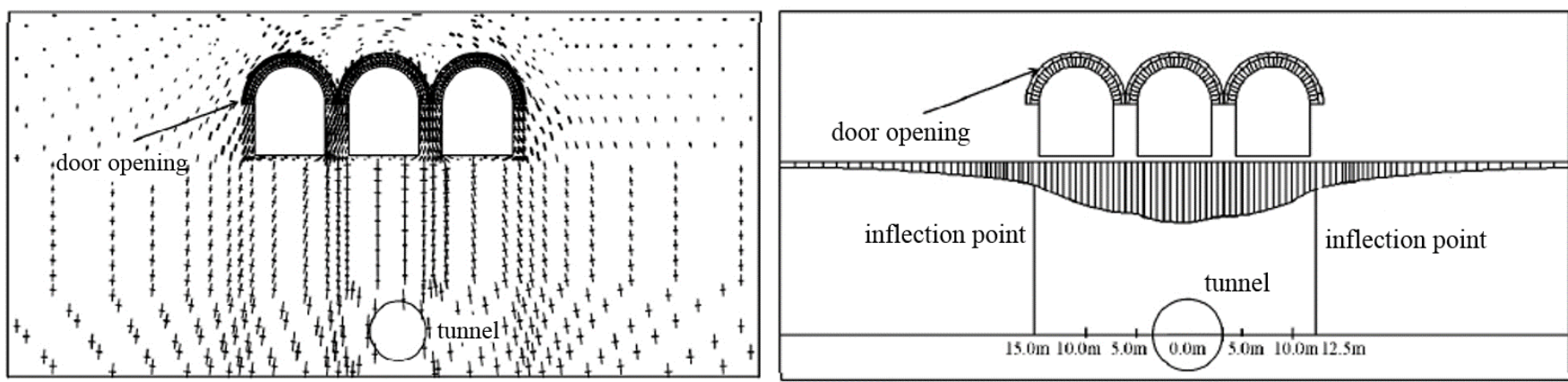

图 2.（a）应力矢量图;

（b）沉降槽矢量图
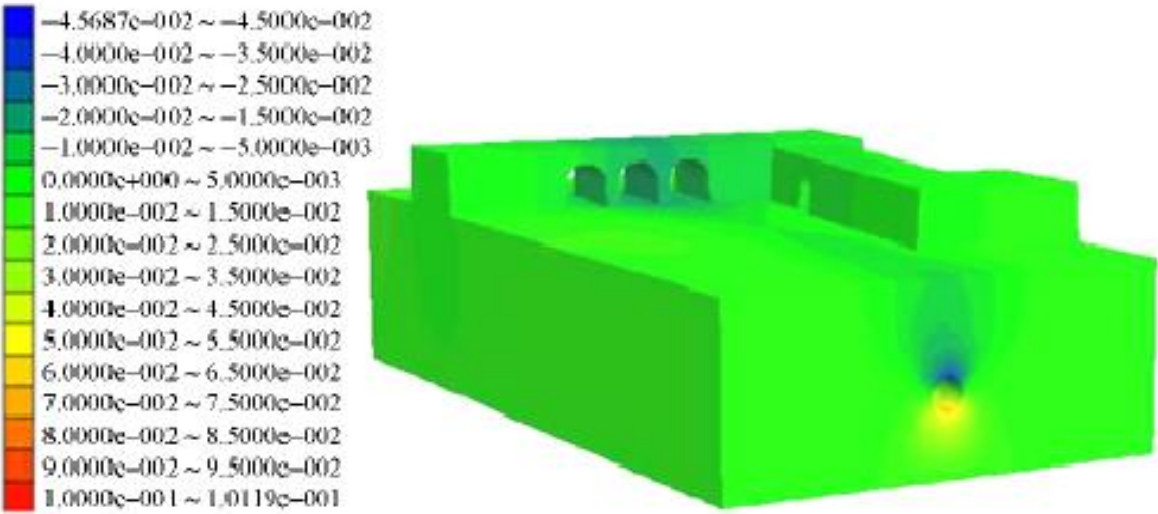

图 3. 沉降变形云图

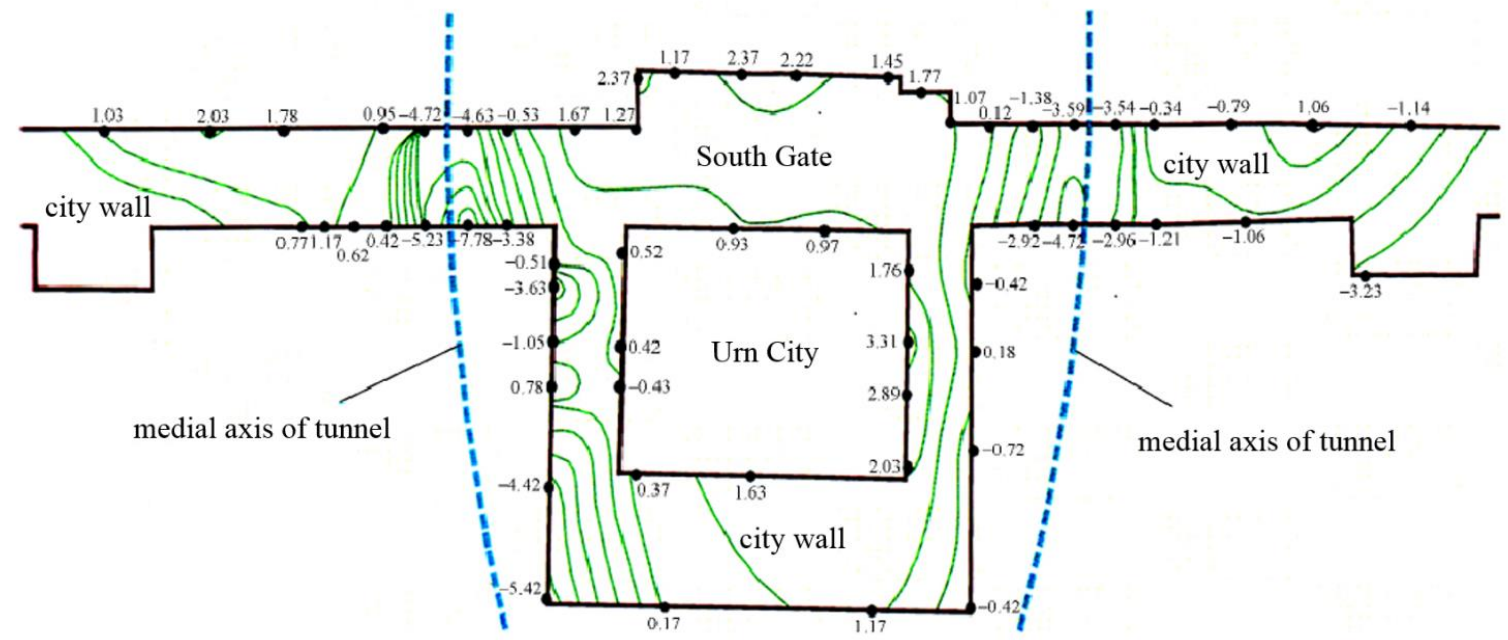

图 4. 南门沉降与等值线 
计算结果表明, 南门附近最大沉降量为 $10 \mathrm{~mm}$, 墙 本身最大沉降量为 $4 \mathrm{~mm}$, 沉降槽宽度接近 $30 \mathrm{~m}$, 最大 坡度不大于 0.001 , 采用优化的防治措施, 如加宽南门 周边半径 350 米, 隧道埋深 17.4 米 18.5 米, 加强了城 墙基础。计算结果满足沉降变形控制标准, 限制在最大 沉降值和倾斜值范围内。

\section{（三）试验结果及比较}

现场监测数据表明, 南、北门城墙变形范围在 $-9.99 \mathrm{~mm}+1.21 \mathrm{~mm}$ 之间, 地表变形范围在 $-7.78 \mathrm{~mm}+3.31 \mathrm{~mm}$ 之间, 均限制在变形阈值范围内, 保 证了地下洞室开挖时闸门的静止和完好。好的。南门沉 降和等值线如图 4 所示。

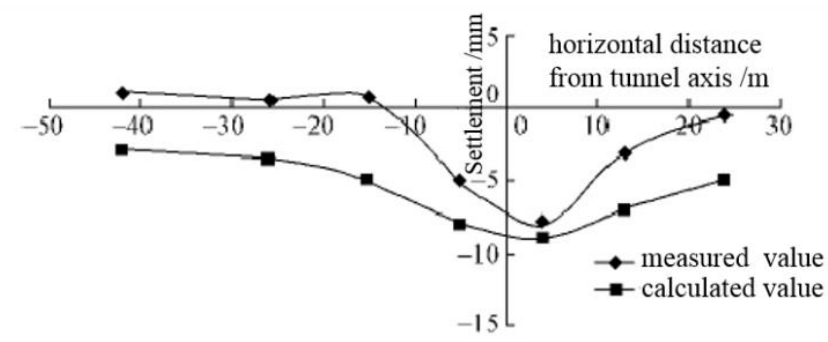

图 5. 数值预测与实测变形图

将数值计算结果与现场实测结果进行了比较 (图 5)。 很明显, 测量值与计算值基本一致, 但略小于计算值。

III. 运营阶段对文物的影响

地铁在运营过程中会对在隧道中产生振动, 车辆振 动是在半封闭的空间中产生的, 在那里车轮在高速前进
的轨道上施加压力。由钢轨冲击载荷、接触面不光滑、 车轮不均匀磨损、偏心安装装置、枕木支撑刚度变化等 因素引起。

振动以波的形式依次通过轨道、扣件、枕木、道碴、 隧道结构、地层向地表传播, 最终影响周围建筑物。根 据土的性质、振动频率和能量以及体波效应, 振动将持 续一段时间。

万松老人塔是北京市重点文物保护单位, 距今已有 700 多年的历史。该塔位于北京市西城区西四南大街 41 号, 砖塔巷以其命名。宝塔最初建于元朝初年, 呈八角 形、七层高, 并于清乾隆十八年 (1753 年) 被提高到九 层。元塔被清代的塔包裹着。是北京市区仅存的砖塔, 古朴典雅、精致。万松老人塔是一座金元风格的八角九 层砖塔, 高约 16 米。院子东西长 14 米, 南北宽 7 米。 面积 99.3 平方米, 其中建筑面积 22.5 平方米。目前, 该塔已向东倾斜约 3 度。

\section{（一）振动控制规范}

北京地铁 4 号线进入万松老人塔文物保护区，中心 线距塔底仅 9 米。根据《万松宝塔现状评估报告》，塔 基基础最终沉降变形的允许值范围为 $+10 \mathrm{~mm} \sim-15 \mathrm{~mm}$ 。 最大沉降差为 $5 \mathrm{~mm}$ 。在理论计算、工程类比和现场调查 的基础上, 提出了控制标准。因此, 修改后的控制标准 为: 塔基础最终整体沉降变形的允许值在 $+10 \mathrm{~mm}$ 和 $-10 \mathrm{~mm}$ 之间变化, 地基最大不均匀沉降小于或等于 $5 \mathrm{~mm}$ 。

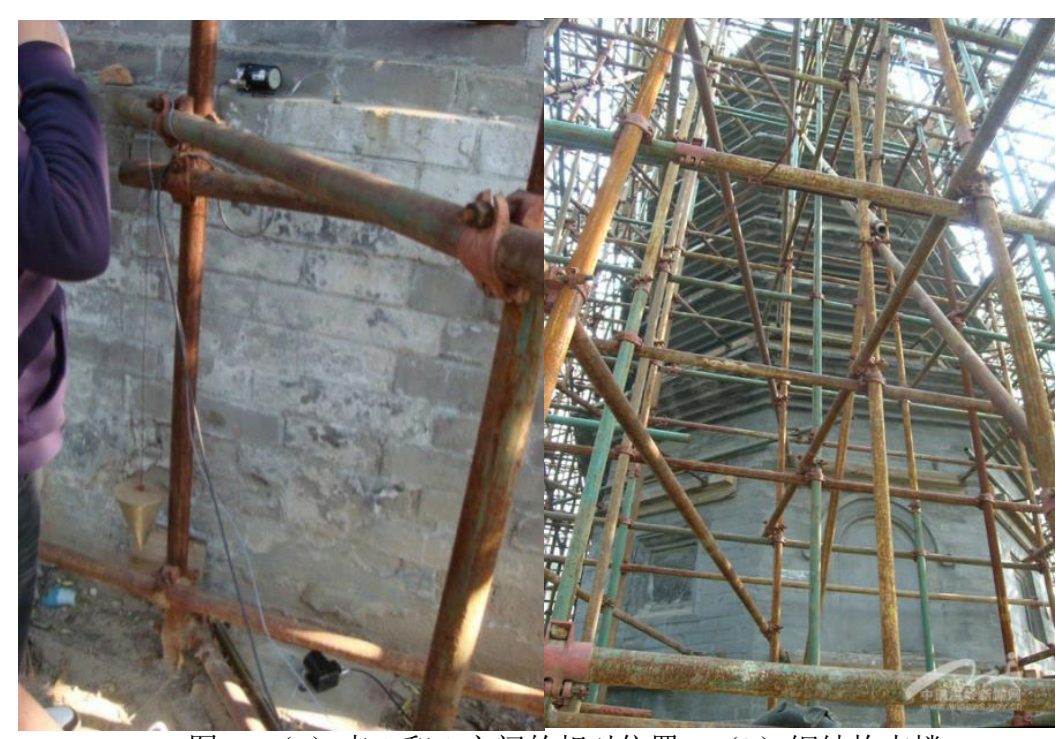

图 6.（a）点 2 和 3 之间的相对位置; （b）钢结构支撑

（二）现场监测结果

为保证施工安全, 采取临时防护措施, 严格执行施 工方案, 如监测施工期间挖出的土方量, 加强灌浆和二 次补充, 保证盾构机在下部顺利通过。在不同的位置指 定了四个测量点, 布设点见表 1 , 具体措施如图 6 所示。

监测数据表明, 除其它常规技术参数外, 塔架在运 行阶段沉降控制在 4.6mm, 远小于临界值 $10 \mathrm{~mm}$ 。所以,
目前的万松尊塔在理论上是安全的。而由于地下地铁的 存在, 采用了附加的钢架支撑对塔身进行加固。同时, 该段地铁采用梯形轨枕, 在一定程度上降低了振动。监 测结果表明, 这种轨枕投入使用后, 地铁引起的水平振 动最大值小于或等于地面交通引起的水平振动最大值, 也就是说, 万松古塔主要受地面交通引起的振动影响。 由于这些措施, 交通工具而不是地铁大大减少了地下交 通。 


\begin{tabular}{cc} 
& 表 1. 测点分布 \\
\hline 点号 & 位置 \\
\hline 1 & 东侧地面 \\
2 & 西侧地面 \\
3 & 塔身西侧地上 $1.4 \mathrm{~m}$ \\
4 & 六层塔顶 \\
\hline
\end{tabular}

\section{IV. 结论与政策建议}

(1) 我国许多城市, 特别是一些拥有悠久历史的城市, 存在许多的文物建筑与地下设施的修建相互影响;

(2) 新建地铁对文物建筑的主要影响是施工过程中的 不均匀沉降和运营过程中的振动问题;

（3）盾构地铁在修建过程中会对地面产生沉降影响, 且沉降量与地铁埋深和工程地质分布有密切关系;

（4）地铁在修建过程中对地表产生的不均匀沉降是对 文物建筑产生不良影响的主要因素, 施工过程中应密切 关注地表沉降量的变化大小以及分布特征;

(5) 为保护文物建筑, 相关部门制定了一系列标准要 求, 这些标准的计算方法和工程要求大多是保守的, 通 常可以保证文物结构的安全;

（6）减少地铁对文物的干扰的主要措施是地铁线路避 绕和文物结构加固; 前者通过主动回避, 减少地铁线路 对文物的干扰; 后一种措施是在地铁线路难以避开时, 通过工程措施对结构或土壤进行加固。提高了文物的土 壤力学性能和抗被动性。

\section{致谢}

本研究受国家自然科学基金: 农户生产联结（PPL） 机制及其关联性信用风险演化机理研究 (71873101) 和 农村金融联结机制及其关联性信用风险演化机理研究 (71373207)联合资助。

\section{参考文献}

[1] 肖金亮, 中国历史建筑保护科学体系的建立与方法论研究. 北京: 清华大学, 2009.

[2] 苗永杰, 盾构法地铁隧道施工过程古建筑保护措施研究. 西安: 长 安大学, 2017.

[3] 雷永生, "西安地铁二号线下穿城墙及钟楼保护措施研究", 岩土力 学, vol. 31, issue 1, pp. 223-228+236, 2010.

[4] 杨开忠, 西安地铁建设中的古建筑保护研究. 北京: 中国地质大学 (北京), 2009.

[5] 狄文莉, 西安地铁五号线之文物影响评估初探. 西安: 西安建筑科 技大学, 2013.

[6] 常海青, 西安城市轨道交通规划文物影响评估研究. 西安: 西安建 筑科技大学, 2013.

[7] 王帆, 姚海波, 胡建林, "浅埋大断面公路黄土隧道下穿古文物遗 址沉降监测与控制", 工业建筑, vol. 44, issue s1, pp. 711-714, 2014.

[8] 颜治国, 西安地铁黄土地层中盾构隧道地表沉降控制理论与技术. 北京: 中国矿业大学(北京), 2012.

[9] 钟铮, "上海外滩文物保护建筑在双侧开挖情况下的沉降变形分析", 建筑施工, vol. 32, issue 11, pp. 1110-1113, 2010.

[10] 枈江飞, 周晓茗, 张吉, 刘振雯, 李宁, "软土地区深基坑开挖对 周边文物建筑沉降的影响", 施工技术, vol. 44, issue 1, pp. 28-31+98, 2015.

[11] 钱春宇, 李俊连, 张炜, 郑建国, 康佐, 高术孝, "地铁施工与运营 振动对文物建筑影响研究", 建筑结构, vol. 45, issue 19, pp. 87-91, 2015.

[12] 孟昭博, 西安钟楼的交通振动响应分析及评估. 西安: 西安建筑 科技大学, 2009 .
[13] 贾颖绚, 郭猛, 刘维宁, 张新金, 刘卫丰, "列车振动荷载对古建 筑的动力影响", 北京交通大学学报, vol. 33, issue 1, pp. 118-122, 2009.

[14] J. S. Yang, B. C. Liu, and M. C. Wang, "Modeling of tunneling-induced ground surface movements using stochastic medium theory", Tunnelling \& Underground Space Technology Incorporating Trenchless Technology Research, vol. 19, issue 2, pp. 113-123, 2004.

[15] I. Ocak, "Interaction of longitudinal surface settlements for twin tunnels in;shallow and soft soils: the case of Istanbul Metro", Environmental Earth Sciences, vol. 69, issue 5, pp. 1673-1683, 2013.

[16] A. K. Valizadeh, M. H. Sadaghiani, and M. M. Ahmadi, "Numerical modeling of ground settlement control of large span underground metro station in Tehran Metro using Central Beam Column (CBC) structure", Tunnelling \& Underground Space Technology Incorporating Trenchless Technology Research, vol. 28, issue 1, pp. $1-9,2012$.

[17] X. Xie, and Y. Yang, M. Ji, "Analysis of ground surface settlement induced by the construction of a large-diameter shield-driven tunnel in Shanghai, China", Tunnelling \& Underground Space Technology Incorporating Trenchless Technology Research, vol. 51, issue pp. 120-132, 2016.

[18] M. Migliazza, M. Chiorboli, and G. P. Giani, "Comparison of analytical method, 3D finite element model with experimental subsidence measurements resulting from the extension of the Milan underground", Computers \& Geotechnics, vol. 36, issue 1, pp. 113-124, 2009.

[19] V. Krylov, and C. Ferguson, "Calculation of low-frequency ground vibrations from railway trains", Applied Acoustics, vol. 42, issue 3, pp. 199-213, 1994.

[20] T. Real, C. Zamorano, F. Ribes, and J. I. Real, "Train-induced vibration prediction in tunnels using 2D and 3D FEM models in time domain", Tunnelling \& Underground Space Technology Incorporating Trenchless Technology Research, vol. 49, issue pp. 376-383, 2015.

[21] L. HALL, "Simulations and analyses of train-induced ground vibrations in finite element models", Soil Dynamics \& Earthquake Engineering, vol. 23, issue 5, pp. 403-413, 2003.

[22] 韩煊, 李宁, and J. R. Standing, "Peck 公式在我国隧道施工地面变 形预测中的适用性分析", 岩土力学, vol. 28, issue 1, pp. 23-28+35, 2007

[23] J. F. Liu, T. Y. Qi, and Z. R. Wu, "Analysis of ground movement due to metro station driven with enlarging shield tunnels under building and its parameter sensitivity analysis", Tunnelling \& Underground Space Technology Incorporating Trenchless Technology Research, vol. 28, issue 3, pp. 287-296, 2012. 